\title{
Ray-Based Statistical Propagation Modeling for Indoor Corridor Scenarios at 15 GHz
}

\author{
Qi Wang, ${ }^{1}$ Bo Ai, ${ }^{1}$ Ke Guan, ${ }^{1}$ David W. Matolak, ${ }^{2}$ Ruisi He, ${ }^{1}$ and Xin Zhou $^{3}$ \\ ${ }^{1}$ State Key Laboratory of Rail Traffic Control and Safety, Beijing Jiaotong University, Beijing 100044, China \\ ${ }^{2}$ Department of Electrical Engineering, University of South Carolina, Columbia, SC 29208, USA \\ ${ }^{3}$ National Institute of Metrology, Beijing 100013, China \\ Correspondence should be addressed to Bo Ai; boai@bjtu.edu.cn
}

Received 1 November 2015; Revised 6 March 2016; Accepted 24 March 2016

Academic Editor: Larbi Talbi

Copyright (C) 2016 Qi Wang et al. This is an open access article distributed under the Creative Commons Attribution License, which permits unrestricted use, distribution, and reproduction in any medium, provided the original work is properly cited.

\begin{abstract}
According to the demands for fifth-generation (5G) communication systems, high frequency bands (above $6 \mathrm{GHz}$ ) need to be adopted to provide additional spectrum. This paper investigates the characteristics of indoor corridor channels at $15 \mathrm{GHz}$. Channel measurements with a vector network analyzer in two corridors were conducted. Based on a ray-optical approach, a deterministic channel model covering both antenna and propagation characteristic is presented. The channel model is evaluated by comparing simulated results of received power and root mean square delay spread with the corresponding measurements. By removing the impact of directional antennas from the transmitter and receiver, a path loss model as well as small-scale fading properties for typical corridors is presented based on the generated samples from the deterministic model. Results show that the standard deviation of path loss variation is related to the Tx height, and placing the Tx closer to the ceiling leads to a smaller fluctuation of path loss.
\end{abstract}

\section{Introduction}

Candidates for the fifth-generation (5G) terrestrial wireless technology have attracted a lot of interest in recent years as the next set of mobile communication standards beyond the $4 \mathrm{G}$ standards $[1,2]$. Although $5 \mathrm{G}$ is not an official term yet, the standardization of $5 \mathrm{G}$ technologies is expected to be finished between 2016 and 2018 [3]. Beyond the capabilities of previous generation systems, the $5 \mathrm{G}$ mobile communications systems will emerge to meet additional demands $[4,5]$. The increase of global mobile data traffic is expected to be more than a factor of 200 from 2010 to 2020, with an even higher growth factor in developed cities and hotspots. However, due to limited available spectrum in the microwave bands, the increasing demand cannot be fully met [6]. So, with much larger required bandwidths, high frequency bands (above $6 \mathrm{GHz}$ ) should be adopted for $5 \mathrm{G}$ mobile systems to support anticipated higher data rates [7-9].

Since a large attenuation is suffered at higher frequencies, the frequency bands with relatively large bandwidth and yet still "low" central frequency are most promising. As one of the candidate frequency bands for $5 \mathrm{G}$ system, the $15 \mathrm{GHz}$ band still has more than $0.7 \mathrm{GHz}$ bandwidth to be allocated [9], which makes this band one of the most promising candidate potential high frequency bands. Furthermore, the $15 \mathrm{GHz}$ band belongs to the $\mathrm{Ku}$-band which is from $12 \mathrm{GHz}$ to $18 \mathrm{GHz}$. The most widespread usage of the Ku-band is traditional satellite mobile services, and only a few publications have appeared on its usage for terrestrial applications [10]. In [11], measurements of the land mobile satellite (LMS) channel are presented. A statistical model for the LMS channel is proposed to show the properties at Ku-band [12]. In [13], both the narrow and wideband parameters were investigated for LMS land at Ku-band. A comparison of the first- and secondorder statistics of the LMS channel at Ku-band and other high frequency bands is presented in [14]. The above publications provide the basic (outdoor) channel properties at Ku-band. However, in this band, channel measurement and modeling have been investigated much less thoroughly than in other frequency bands, and most of the investigations are limited to LMS channel conditions. Measurements of an indoor radio frequency (RF) propagation channel were performed at $14 \mathrm{GHz}$ in [15]. However, only some initial measurement results were presented without in-depth analysis or modeling. 
The research on propagation for indoor corridor scenarios has drawn significant attention over the years. For measurement and modeling, statistical and deterministic channel model methods are commonly used. The statistical method mainly pertains to the measurement-based modeling [16]. This method provides the large- and small-scale fading properties which are extracted from measurement results [17]. In [18], the path loss exponent, standard deviation of the large-scale fading, and Ricean $K$-factor were extracted and analyzed in three different corridors at $450 \mathrm{MHz}, 900 \mathrm{MHz}$, $1.35 \mathrm{GHz}$, and $1.89 \mathrm{GHz}$. In [19, 20], MIMO channel measurements for four typical indoor scenarios including the corridor were made at $1.9 \mathrm{GHz}$, and correlations between the channel's $K$-factor, delay spread, and shadowing were derived. A dynamic propagation measurement in a corridor at $5.2 \mathrm{GHz}$ with a moving transmitting antenna was performed in [21]. At a millimeter wave band of $60 \mathrm{GHz}$, statistical results and channel models extracted by impulse response measurements for an indoor scenario including offices and corridors were presented in [22]. None of these works considered Ku-band, and none considered the influence of the antenna height.

The deterministic modeling method mainly refers to deterministic channel modeling based on a ray-optical approach. This method can provide accurate modeling of the environment and wave propagation, at the expense of a relatively high computational complexity, and inherent site-specificity. In [23], a comparison of measurements in an indoor corridor at $900 \mathrm{MHz}$ and $1800 \mathrm{MHz}$ and 2D ray tracing simulations were presented. In [24], a 3D ray tracing method was used to evaluate a multiple-input multipleoutput (MIMO) system in an indoor rectangular corridor at $2.53 \mathrm{GHz}$. In [25], the path loss in three corridors was modeled by a deterministic method using ray tracing, but the resulting mean square error (MSE) values between predictions and measurements were all above $6.5 \mathrm{~dB}$. At a millimeter wave band of $60 \mathrm{GHz}$, the channel characteristics in an indoor corridor were addressed in [26] using ray tracing and an ITU-R model [27]. Generally, the accuracy of deterministic channel model still needs to be improved with low complexity for indoor corridor scenarios, and, as an important part of this channel model, the antenna characteristics also should be considered.

To fill these gaps, the main contribution of this paper is to present a statistical path loss model and small-scale fading properties for indoor corridor scenarios based on a rayoptical simulator validated by measurements in the $15 \mathrm{GHz}$ band. By analyzing the influence of directional antennas and multipath components, the correlation between the height of transmitter $(\mathrm{Tx})$ and the standard deviation of shadow fading is modeled based on extensive simulations. With the aid of this path loss model for a typical corridor, the path loss can be predicted to achieve sufficient accuracy for the design and planning of various communication systems at $15 \mathrm{GHz}$.

This paper is organized as follows. The indoor corridor measurement is described in Section 2. Section 3 provides a detailed deterministic propagation modeling approach. The validation of the simulation is presented in Section 4. In Section 5, the analysis and statistical modeling of the simulation are reported. Section 6 concludes the paper.
TABLE 1: Electromagnetic parameters of building materials.

\begin{tabular}{lcc}
\hline Materials & Relative permittivity & Conductivity $(\mathrm{S} / \mathrm{m})$ \\
\hline Plasterboard wall & 2.94 & 0.0788 \\
Ceiling & 1.50 & 0.0117 \\
Floor & 3.66 & 0.1710 \\
\hline
\end{tabular}

\section{Indoor Corridor Measurement}

2.1. Measurement Locations. Measurements were performed at two different floors of the Mechanical Engineering Building in Beijing Jiaotong University, China. The Mechanical Engineering Building is a 10-floor office building with all the classrooms on the west side and all the faculty offices on the east side. The measurements were carried out on floors 2 and 4 of the east side in the Mechanical Engineering Building, as shown in Figure 1. These two corridors have the same size and the same heights of 2.7 meters. The only difference between these two corridors is that there is an open area on floor 2 as marked in Figure 1.

The fixed Tx with a single antenna is located at center of the corridor, and there are several line-of-sight (LoS) locations of the receiver $(\mathrm{Rx})$ located at the center or nearer one side of the corridor, 1.2 meters apart from each other, as marked in Figures 1(a) and 1(b). The smallest Tx-Rx distance is also 1.2 meters, and the largest distance is about $22 \mathrm{~m}$. Acting as a single-antenna user, the Rx's antenna is moved along a straight line. The measurements are performed in controlled indoor corridor environments, where there were no persons or movements on either of the two floors. The corridor surface materials are plasterboard "drywall" for the walls, ceramic covered by floor tiles on the floors, and acoustic ceiling tiles for the ceiling, which are very common in most buildings. The electromagnetic parameters including permittivity and conductivity of these typical building materials are provided in an ITU-R recommendation for indoor scenarios [28], and these are calculated and listed in Table 1.

2.2. Measurement System. The measurements were carried out at the center frequency of $15 \mathrm{GHz}$. The channel between single Tx and single $\mathrm{Rx}$ of each location was measured by a vector network analyzer (VNA) with a bandwidth of $1 \mathrm{GHz}$ ranging from $14.5 \mathrm{GHz}$ to $15.5 \mathrm{GHz}$ using 1024 frequency points, yielding a time resolution of $1 \mathrm{~ns}$. The transmitted power is $0 \mathrm{dBm}$. The dynamic range of the VNA is $85 \mathrm{~dB}$ with a noise floor of $-88 \mathrm{dBm}$. At each $\mathrm{Rx}$ position, the measurement was conducted twice for time averaging, and totally five different positions were measured for spatial averaging of small-scale effects: these positions were separated by approximately two wavelengths. The arrangement of these positions is shown in Figure 2. Both the $\mathrm{Tx}$ and $\mathrm{Rx}$ were equipped with directional antennas designed by a company named "Ocean Microwave" with the model number of "OBH08180," which are ultrawideband (UWB) horn antennas with a gain of about $13.5 \mathrm{dBi}$ at $15 \mathrm{GHz}$, as shown in Figure 3(a). Figure 3(b) shows the antenna pattern of OBH08180, where the E plane and $H$ plane represent the vertical cut and horizontal cut of the antenna, respectively. Both the $\mathrm{Tx}$ and $\mathrm{Rx}$ antennas 


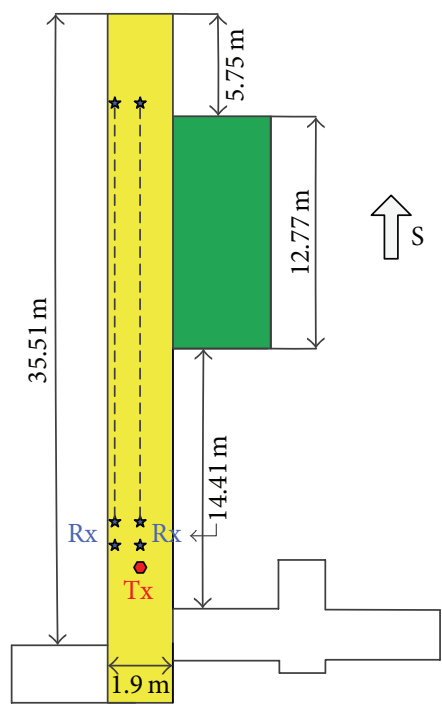

Open area

Main corridor

(a)

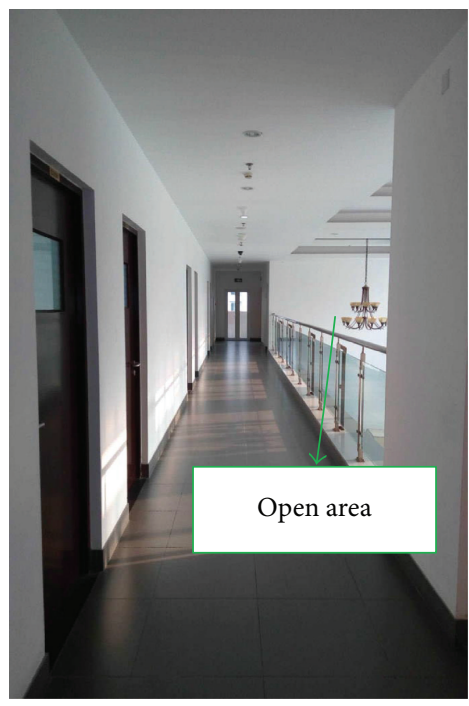

(c)

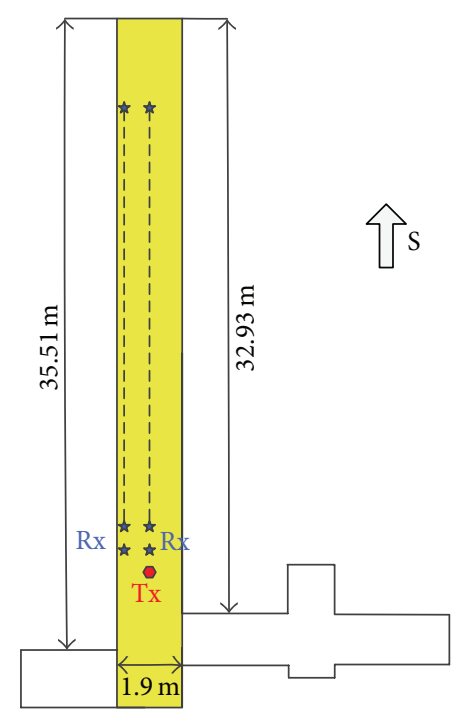

Main corridor

(b)

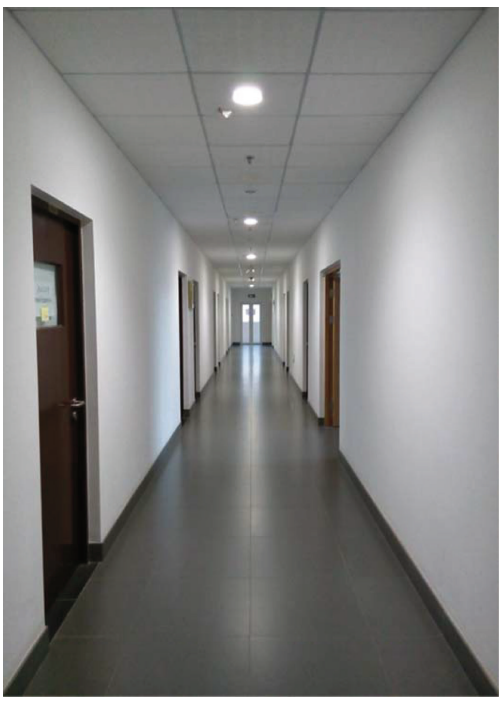

(d)

Figure 1: (a) Diagram of floor 2 in Mechanical Engineering Building. (b) Diagram of floor 4 in Mechanical Engineering Building. (c) Photograph of measurement scenario on floor 2. (d) Photograph of measurement scenario on floor 4.

were set up on wooden tripods, paralleling the corridors for all the measurements. Between the Tx/Rx and VNA, we used a long cable, and the attenuation caused by the cable was calibrated out for measurements. Figure 4 illustrates the whole measurement system. All the measurement parameters are listed in Table 2.

\section{Deterministic Propagation Modeling}

The radio channel behavior of this indoor corridor scenario has been simulated by a deterministic propagation model, that is, the ray-optical approach. As a deterministic model, this self-developed 3D method includes the direct path, specular reflection paths, and scattering paths. Based on image method, specular reflection paths can be computed by up to the 5th order in our simulator. Scattering paths are those on surfaces which can be seen by both the Tx and Rx. These surfaces are segmented into tiles and only one-order scattering paths are computed according to radar equation for scattering processes [29]. The 3D ray-optical model consists of the antenna modeling, modeling of the environment, and the 3D ray-based channel modeling including the polarization [30].

3.1. Antenna Modeling. The antenna gain, polarization, and elevation and azimuth angles strongly affect the propagation 
TABLE 2: Measurement parameters.

\begin{tabular}{lc}
\hline Parameters & Value \\
\hline Center frequency & $15 \mathrm{GHz}$ \\
Bandwidth & $1 \mathrm{GHz}$ \\
Frequency points & 1024 \\
Time resolution & $1 \mathrm{~ns}$ \\
Transmitted power & $0 \mathrm{dBm}$ \\
Dynamic range & $85 \mathrm{~dB}$ \\
Noise floor & $-88 \mathrm{dBm}$ \\
Antenna gain & $13.5 \mathrm{~dB}$ \\
\hline
\end{tabular}

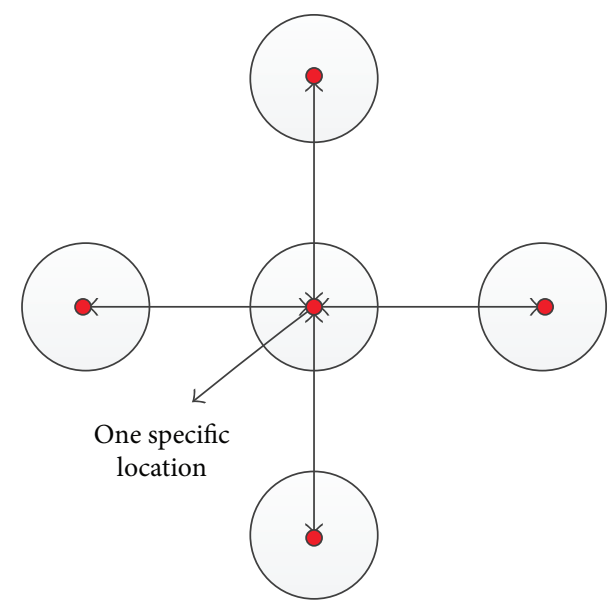

Figure 2: Positions of $\mathrm{Rx}$ at each location. One received point is at the center of one specific location, surrounded by the other four points.

[31]. Therefore, especially for a directional antenna in an indoor scenario, the antenna should be modeled precisely, and both the gain and the polarization of the antenna need to be accounted for. For the gain, two orthogonal components of the horizontal and the vertical fields of the antenna patterns are defined as $E_{\varphi}$ and $E_{\vartheta}$, respectively. The 2D antenna patterns of $E_{\varphi}$ and $E_{\vartheta}$ are obtained at all angles of departure or arrival by using the linear interpolation, as showed in Figures 5(a) and 5(b). Then the 3D antenna pattern can be generated by the interpolation of the $2 \mathrm{D}$ patterns, as shown in Figure 5(c).

3.2. Modeling of the Scenario. As a deterministic model, the modeling of the scenario influences the propagation significantly due to scenario-dependent propagation mechanisms. The scenario model consists of the structure of the corridors, the electromagnetic parameters (permittivity and conductivity) of all objects, the position of $\mathrm{Tx} / \mathrm{Rx}$, and the directions of the antennas. Figure 6(a) shows the scenario model of floor 2 in the Mechanical Engineering Building, where the red dot and the red line represent the Tx's position and main beam direction, and the blue dots represent the $\mathrm{Rx}$ in different locations, whose directions are always pointing parallel to the corridor.

3.3. 3D Ray-Optical Based Channel Simulation. Using the 3D ray-optical based channel simulation, the information of each ray can be obtained, including type of the path (i.e., direct path, reflection path, or scattering path), path delay, path loss, and $3 \mathrm{D}$ angular properties. A snapshot of the ray tracing result is illustrated in Figure 6(b). Specular reflections are calculated based on the image method [32]. Only the single scattering paths are considered by dividing each surface into small square tiles. By accounting for the antenna gain, the time-variant channel impulse response (CIR) $h(t, \tau)$ of the $3 \mathrm{D}$ ray-optical model can be obtained. It can be expressed in the complex baseband as follows [29]:

$$
h(t, \tau)=\sum_{k=1}^{N(t)} a_{k}(t) e^{-j\left(2 \pi f_{c} \tau_{k}(t)+\varphi_{k}(t)\right)} \delta\left(\tau-\tau_{k}(t)\right),
$$

where $a_{k}(t), \tau_{k}(t)$, and $\varphi_{k}(t)$ represent the amplitude, the delay, and the additional phase shift of the $k$ th multipath component at time $t$ for a specific carrier frequency $f_{c}$, respectively. The amplitude $a_{k}(t)$ and the phase $\varphi_{k}(t)$ can be combined as a complex coefficient $\overline{a_{k}(t)}$ for each multipath component, which can be expressed as

$$
\overline{a_{k}(t)}=\vec{e}_{\mathrm{Rx}}{ }^{H} \cdot \mathbf{P}_{k}(t) \cdot \vec{e}_{\mathrm{Tx}} \cdot L_{k}(t),
$$

where $(\cdot)^{H}$ donates the Hermitian transpose of a matrix. $\vec{e}_{\mathrm{Rx}}$ and $\vec{e}_{\mathrm{Tx}}$ are both the $2 \times 1$ complex polarization vectors for $\mathrm{Rx}$ and Tx antennas, respectively. These vectors include the gain and the polarization of the antennas. By utilizing the Jones calculus in [33], these two vectors can be obtained. $\mathbf{P}_{k}(t)$ is the $2 \times 2$ complex channel polarization matrix including the losses of the considered reflection or scattering path which can be calculated according to [34]. The complex coefficient $L_{k}(t)$ includes the propagation loss and the phase shift of the $k$ th multipath component. For the direct and reflected paths, $L_{k}(t)$ can be obtained according to free space attenuation, expressed as

$$
L_{k}(t)=\frac{\lambda}{4 \pi r_{k}(t)} \cdot e^{-j 2 \pi r_{k}(t) / \lambda}
$$

where $\lambda$ is the wavelength and $r_{k}(t)$ is the total distance of the corresponding path from Tx to Rx. For the scattering paths, the term $L_{k}(t)$ needs to be calculated by the radar equation for scattering processes, shown as

$$
L_{k}(t)=\frac{\lambda \sqrt{A_{k}}}{(4 \pi)^{3 / 2} r_{T_{k}}(t) r_{R_{k}}(t)} \cdot e^{-j 2 \pi\left(r_{T_{k}}(t)+r_{R_{k}}(t)\right) / \lambda},
$$

where $r_{T_{k}}(t)$ and $r_{R_{k}}(t)$ are the distances between the scatterer point of corresponding path to the $\mathrm{Tx}$ and the $\mathrm{Rx}$, respectively. $A_{k}$ is the area of the tile which is the segmentation of each surface that we previously mentioned. More comprehensive details of this ray-optical channel model can be found in [29].

\section{Validation of the Simulation}

4.1. Received Power Comparison. The comparison of received power based on the ray-optical model and measurement is shown in Figure 7, where the corresponding results are 


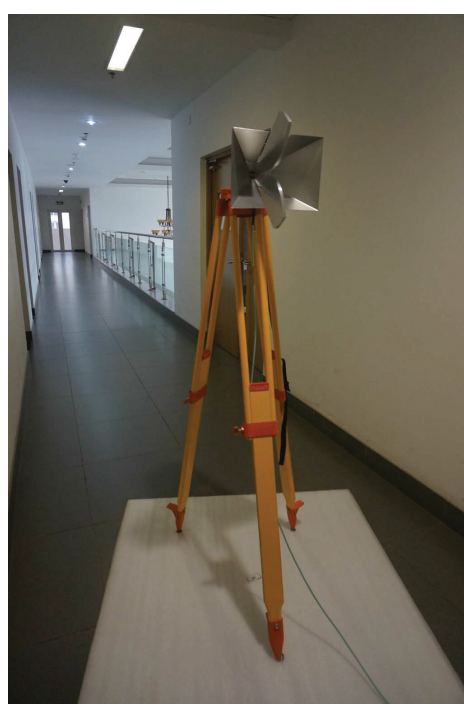

(a)

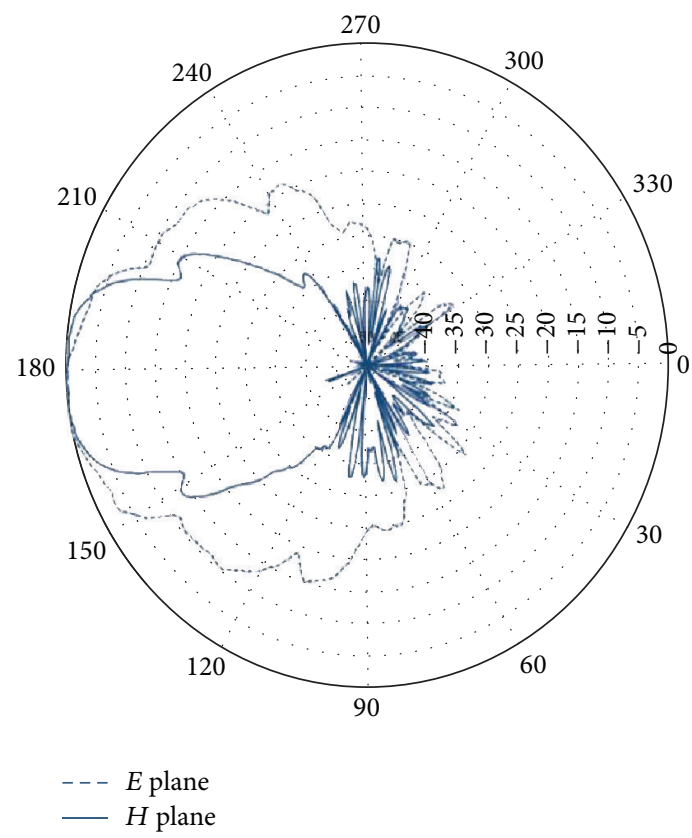

(b)

FIGURE 3: (a) Directional antenna: OBH08180. (b) Antenna pattern of OBH08180.

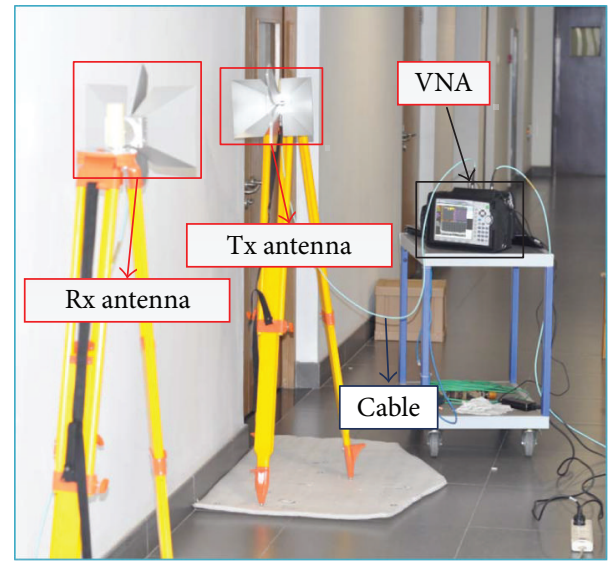

Figure 4: Measurement system with Tx, Rx, VNA, and cable.

obtained from floor 2, with both Tx and Rx located at the center of the corridor at a height of $1.4 \mathrm{~m}$. In Figure 7, the triangles and the dashed black line represent all the original measurement results and the mean value of these measured data points, respectively. The solid black line shows the results simulated by the ray-optical model. The selected position and direction of the antennas can also be found in Figure 6(a). For measurement data, some of the original results that are $10 \mathrm{~dB}$ larger or smaller than the mean value at each Rx's position are removed. With the antenna modeling and the consideration of the cable loss, a good agreement between simulation and measurement can be observed. The mean error (ME), standard deviation (Std), and root mean square error (RMSE) are $1.12 \mathrm{~dB}, 2.23 \mathrm{~dB}$, and $2.44 \mathrm{~dB}$, respectively.
However, some mismatch can also be observed. One reason for the discrepancies between measurements and model is that the objects like office doors and some small signs that have different scattering and reflection properties are not considered in the simulation for the reason of complexity. Figure 7 also demonstrates the predicted results of free space model and two-ray model by considering the directional antennas at both communication ends. From the comparison results, it can be observed that, at the distance smaller than $10 \mathrm{~m}$ from $\mathrm{Tx}$ to $\mathrm{Rx}$, there are almost no differences between these two models and the proposed model; however, at larger distances, the proposed model has the best agreement with the measured data. This is because the directional antennas filter out most of the multipath components; however, as the distance increases from $\mathrm{Tx}$ to $\mathrm{Rx}$, more multipath components can be received from the main lobe of the antennas.

4.2. RMS Delay Spread Comparison. The comparison of the RMS delay spread calculated from simulations and measurements is investigated next. The mean delay is defined as follows:

$$
\bar{\tau}=\frac{\sum_{k} P\left(\tau_{k}\right) \tau_{k}}{\sum_{k} P\left(\tau_{k}\right)},
$$

where $P\left(\tau_{k}\right)$ is the received power at each discrete multipath delay $\tau_{k}$. The RMS delay spread can be calculated as follows [35]:

$$
\sigma_{\tau}=\sqrt{\overline{\tau^{2}}-(\bar{\tau})^{2}},
$$

where $\overline{\tau^{2}}$ can be computed as

$$
\overline{\tau^{2}}=\frac{\sum_{k} P\left(\tau_{k}\right) \tau_{k}^{2}}{\sum_{k} P\left(\tau_{k}\right)} .
$$




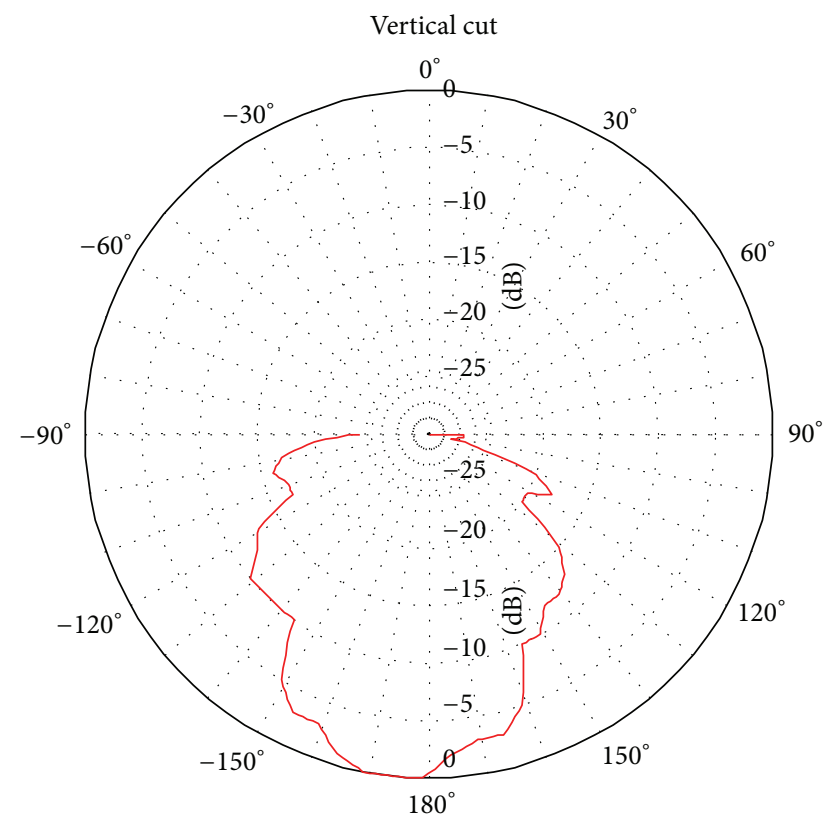

(a)

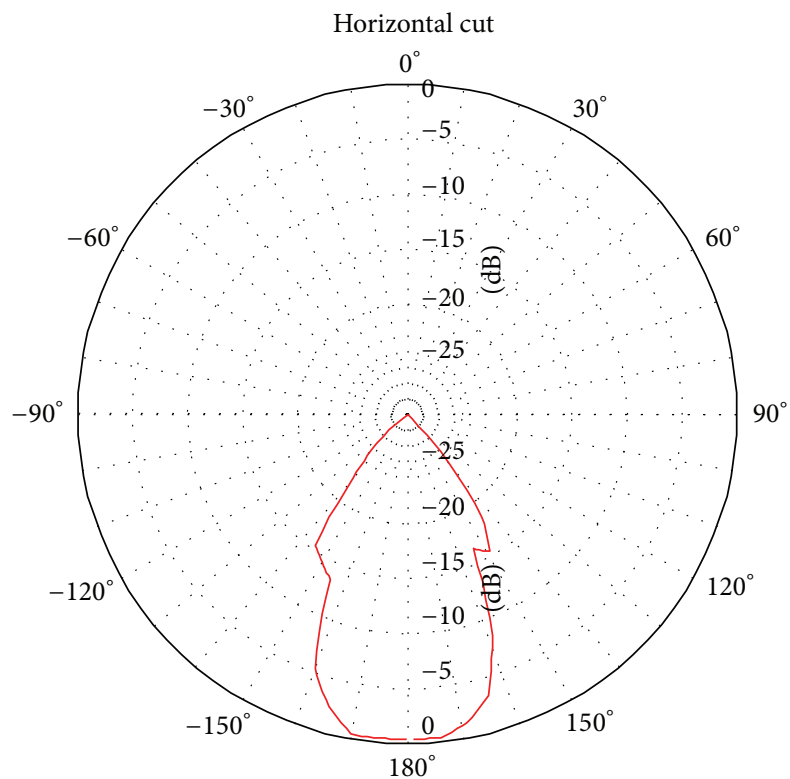

(b)

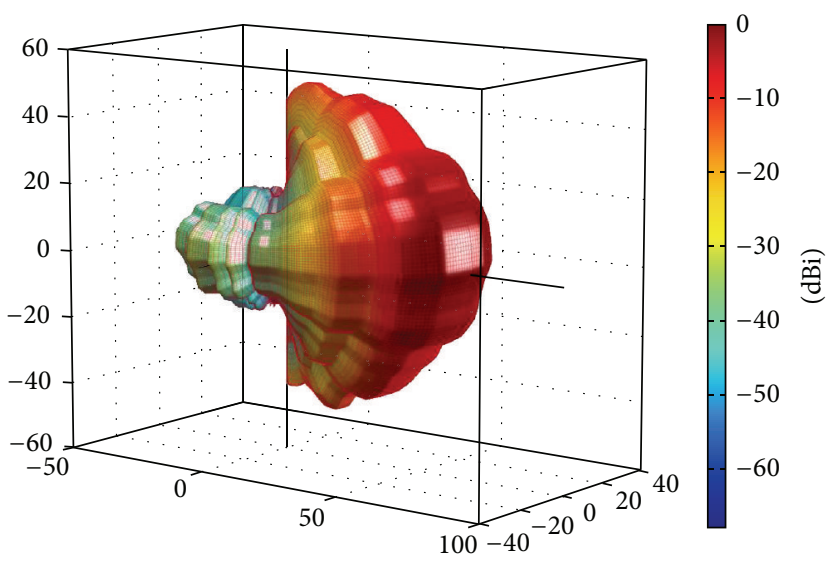

(c)

Figure 5: 3D pattern of the OBH08180 antenna, interpolated by the vertical and horizontal cut.

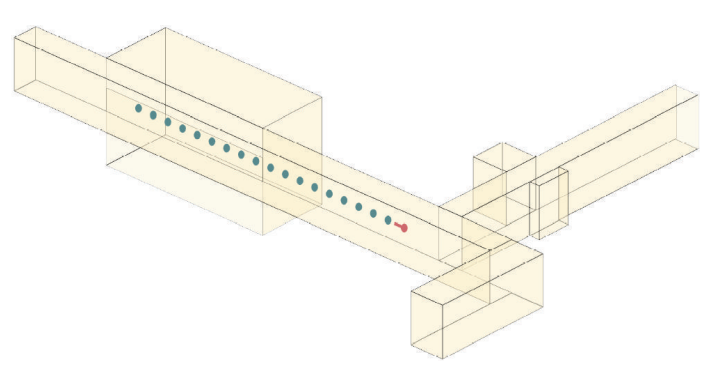

(a)

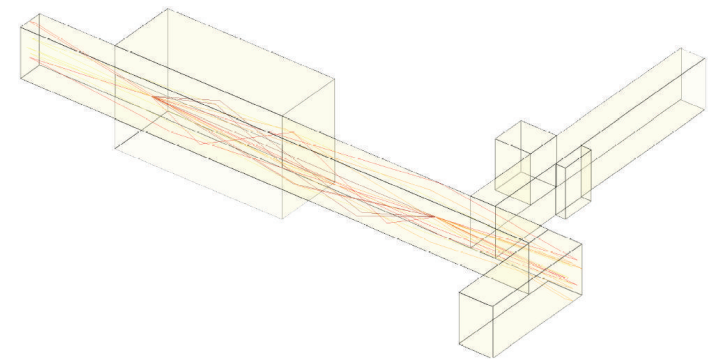

(b)

Figure 6: (a) Scenario model of floor 2 in Mechanical Engineering Building. (b) A snapshot of the ray tracing result.

The bandwidth of the measurement is $1 \mathrm{GHz}$, which can be characterized as a UWB signal. However, only one deterministic frequency point can be simulated in the rayoptical simulation. In order to improve the accuracy of the simulation, the subband divided ray tracing algorithm [36] is used. For this simulation, the entire UWB bandwidth is divided into four subbands, each with a $250 \mathrm{MHz}$ bandwidth. At the center frequency of each subband, the CIR is obtained 


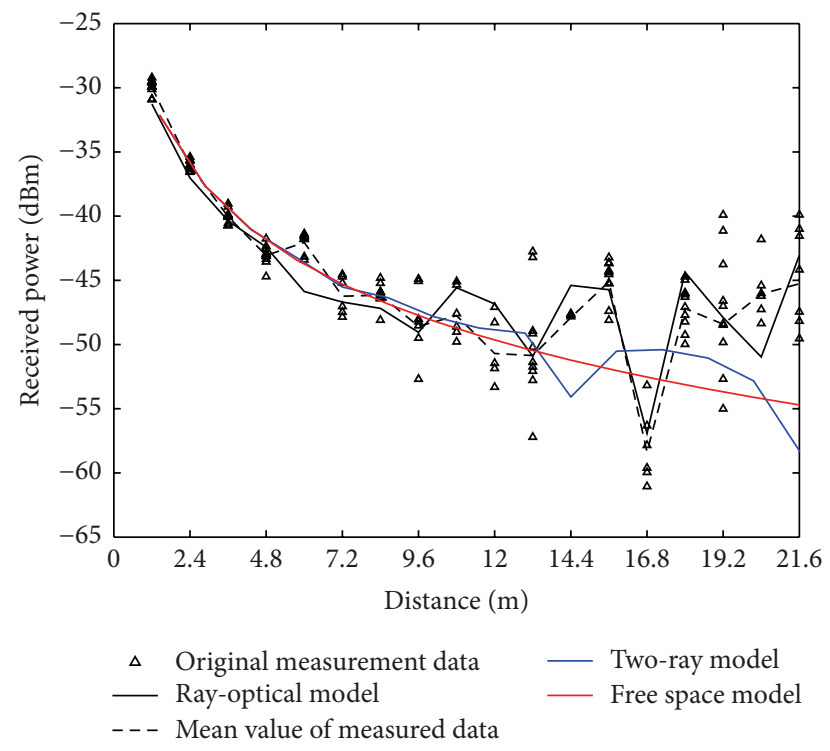

FIGURE 7: Received power versus link distance derived from rayoptical model, free space model, two-ray model, and measurements on floor 2 .

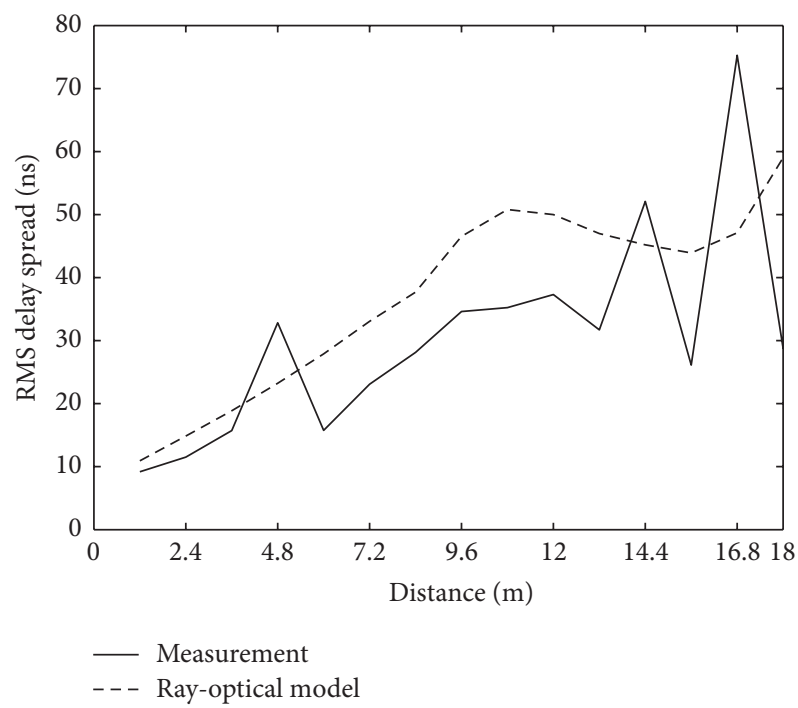

FIGURE 8: RMS delay spread versus link distance derived from rayoptical model and measurement on floor 2.

in delay domain. Then the channel transfer function (CTF) at each subband is obtained by Fourier transform. By concatenating the CTFs of all subbands, the CTF of entire bandwidth is obtained. Finally, the CIR in delay domain for the entire bandwidth is regenerated by the inverse Fourier transform.

In order to get the measured RMS delay spread, a threshold which lies $6 \mathrm{~dB}$ above the noise level is applied [37]. The comparison of RMS delay spread based on ray-optical model and measurement is shown in Figure 8. The characteristics of this figure are summarized as follows:

(1) It can be observed that the RMS delay derived from simulation is of the same order of magnitude as the
TABLE 3: The difference between the measurement and the rayoptical model.

\begin{tabular}{lccc}
\hline Parameters & ME & Std & RMSE \\
\hline Received power on floor 2 & $1.12 \mathrm{~dB}$ & $2.23 \mathrm{~dB}$ & $2.44 \mathrm{~dB}$ \\
Received power on floor 4 & $0.67 \mathrm{~dB}$ & $2.84 \mathrm{~dB}$ & $2.82 \mathrm{~dB}$ \\
RMS delay spread on floor 2 & $6.58 \mathrm{~ns}$ & $13.77 \mathrm{~ns}$ & $14.84 \mathrm{~ns}$ \\
\hline
\end{tabular}

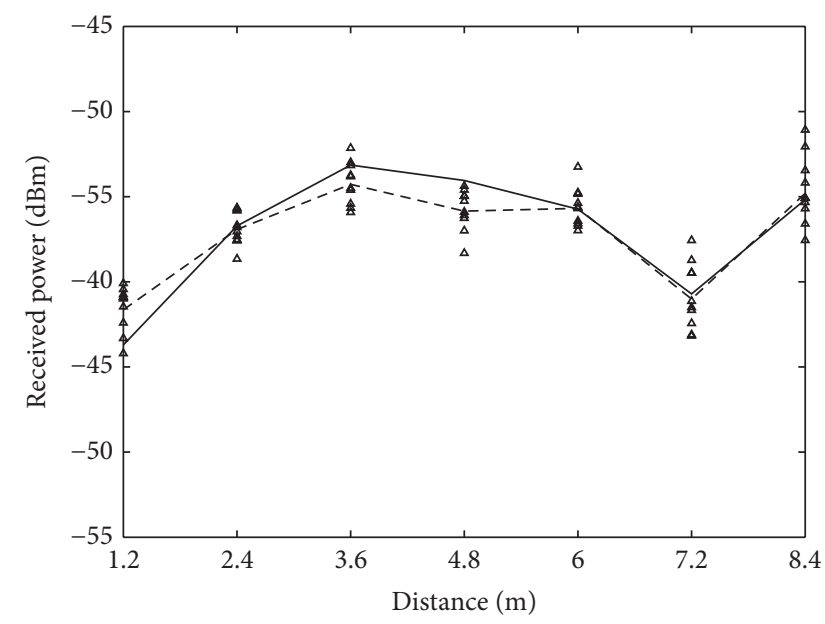

$\Delta$ Original measurement data $\quad$ Ray-optical model - - - Mean value of measured data

FIGURE 9: Received power versus link distance derived from rayoptical model and measurements on floor 2.

one derived from the measurement. The ME, Std, and RMSE are $6.58 \mathrm{~ns}, 13.77 \mathrm{~ns}$, and $14.84 \mathrm{~ns}$, respectively.

(2) However, the simulated RMS delay spread is smoother. This can be explained by the fact that the simulation likely does not model all multipath components, whereas measurements do capture them. The simulated RMS delay spread varies more smoothly than measurements because it inherently must simplify the environment.

(3) For most of the measurement positions, the simulated RMS delay spread is larger than the measured one. This is because, in the simulation results, except the direct path and scattering paths, about $2 / 3$ multipath components are from reflections, and either the number of these multipath components or their strength may be overestimated by adopting the subband divided ray tracing algorithm.

4.3. Validation for Ray-Optical Model. To better use the results to analyze and build a statistical model for large-scale fading, the accuracy of the proposed ray-optical model is evaluated in two other scenarios with different settings.

The first one is still on floor 2, but the Tx and $\mathrm{Rx}$ antennas' heights are $2.45 \mathrm{~m}$ and $1.45 \mathrm{~m}$, respectively. Only seven positions are measured to show the influence of the directional antennas. The comparison of received power based on the ray-optical model and measurement is shown in Figure 9. A good agreement between the simulation and 


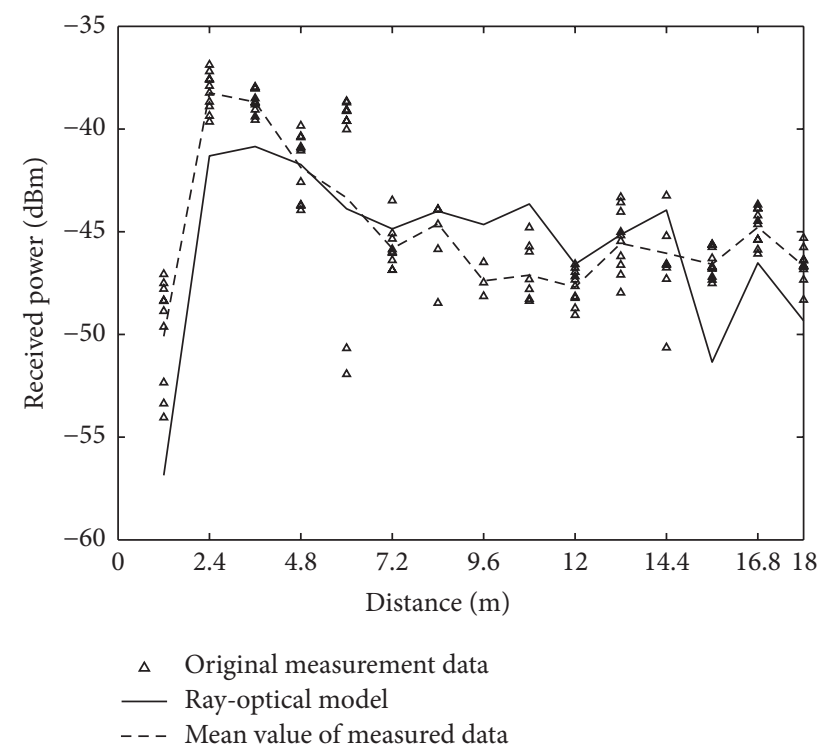

FIGURE 10: Received power versus link distance derived from rayoptical model and measurements on floor 4.

the measurement can be observed and influence of the directional antennas can also be seen at the first three positions, as the received power values are increasing at these positions because of the misalignment of $\mathrm{Tx}$ and $\mathrm{Rx}$. The second comparison is on floor 4 . The heights of the Tx and $\mathrm{Rx}$ antennas are both $1.4 \mathrm{~m}$, but the $\mathrm{Rx}$ is located at the east side of the corridor with a distance of $0.6 \mathrm{~m}$ from the antenna to the wall. In this scenario, the comparison of received power based on the ray-optical model and measurement is shown in Figure 10. The ME, Std, and RMSE are $0.67 \mathrm{~dB}, 2.84 \mathrm{~dB}$, and $2.82 \mathrm{~dB}$, respectively. For different parameters obtained on different floors, the ME, Std, and RMSE of the difference between the measurement and the ray-optical model are summarized in Table 3.

\section{Analysis and Statistic Modeling of the Simulation}

As the deterministic model has been validated in Section 4 by using RMS delay spread and received power which includes both the large- and small-scale fading for indoor corridor scenarios at $15 \mathrm{GHz}$, it is possible to simulate more conditions at this frequency band to show the influence of different antennas, different multipath components, and different Tx heights. Also, the small-scale fading properties can be obtained. In our simulator, the propagation channel model can be coupled with various antenna configurations; that is to say, a pure propagation channel can be obtained without the influence of antennas (or equivalently with isotropic antennas). So, in this section, we also present a statistical path loss model for a general indoor corridor with the same materials for walls, floors, and ceiling as those we measured.

5.1. Influence of the Antenna. The simulation scenario is the corridor which has same dimension and materials with the one on floor 4 . The heights of the $\mathrm{Tx}$ and $\mathrm{Rx}$ antennas are

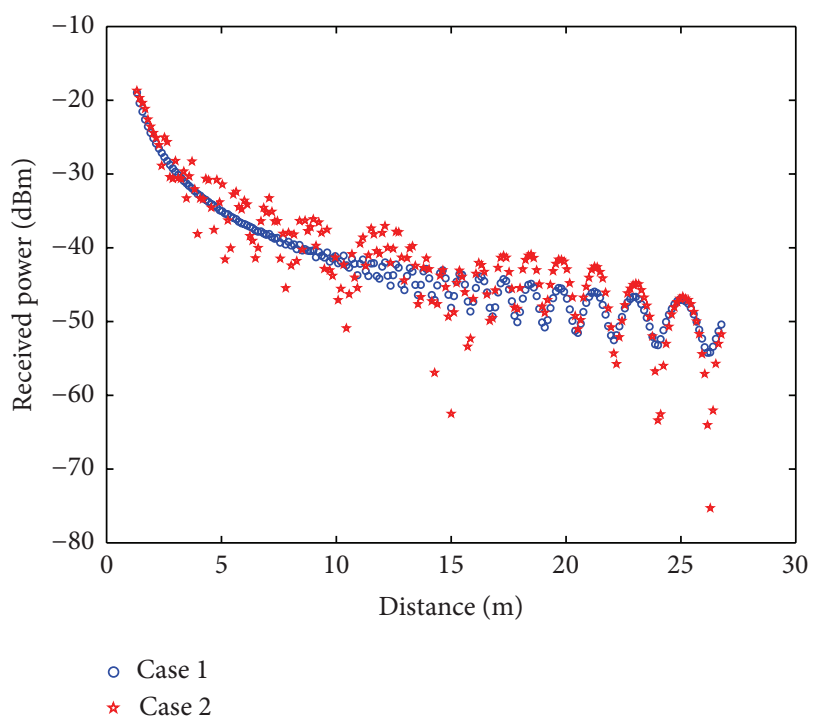

FIGURE 11: Received power versus link distance with different antennas.

both $1.5 \mathrm{~m}$, and both antennas are located at the center of the corridor. For this simulation, two different cases are considered: case 1 is configured with the same directional antennas denoted as OBH08180, while omnidirectional antennas are utilized in case 2 with almost the same gain as the OBH08180. The distance between adjacent locations of Rxs is only $12 \mathrm{~cm}$, and the received power of two cases is shown in Figure 11. From the simulation results, it can be concluded that less fluctuation can be observed in case 1, and the path loss exponent in case 1 is slightly smaller than in case 2 . This can be explained by noting that the directional antennas filter out most of the multipath components, and the LoS component is "strengthened" relatively. To obtain a pure propagation model for indoor corridor scenarios, the influence of antennas should be removed.

5.2. Influence of Different Multipath Components. By removing the impacts of antennas at both link ends, the influence of different multipath components can be investigated. Without loss of generality, a common corridor with a width of $2 \mathrm{~m}$ and a height of $3 \mathrm{~m}$ is generated, and the materials for all the surfaces are the same as those we measured in Section 2. The distance between adjacent locations of Rxs is only $1 \mathrm{~cm}$; more data are thus simulated to reflect both the large- and smallscale fading characteristics.

Figure 12 shows the influence of different multipath components. The blue line is the received power simulated with LoS, scattering paths, and reflection paths where more multiple reflection paths up to 3 rd order are added. The red triangles are the results without multiple reflected paths. The green circles are the results with only LoS and single reflections. In indoor corridor scenarios, it can be observed that the path loss exponent is mainly influenced by the multiple reflection paths; in other words, the exponent becomes much smaller in a traditional log-distance path loss model when all of these multiple reflections are included. The deep fading is mainly caused by the scattering paths according to 


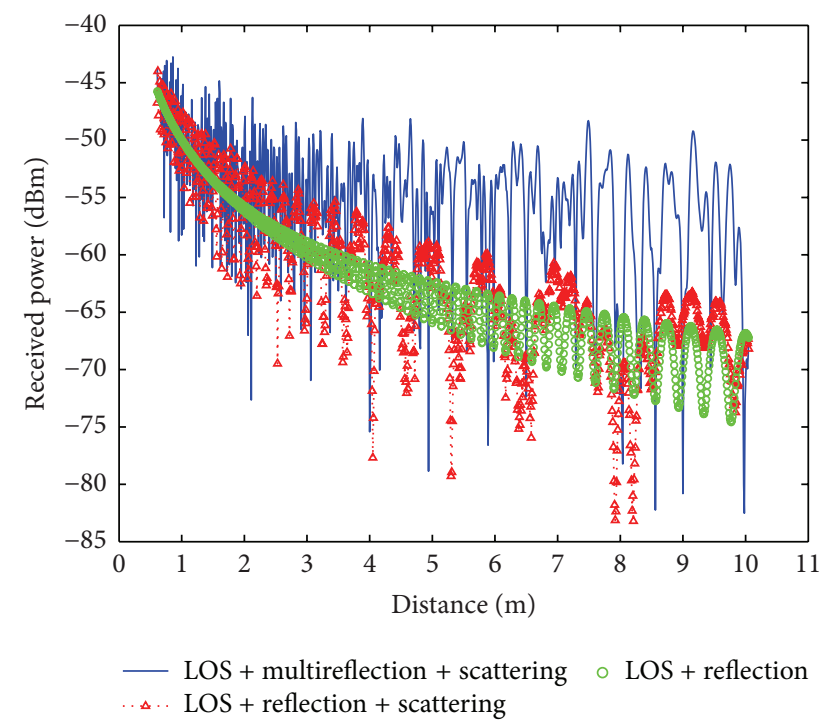

FIGURE 12: Simulated received power versus link distance derived from different multipath components.

the comparison between green circles and red triangles. The fluctuation always exists because of the numerous multipath components at this frequency band.

5.3. Influence of the Tx Height $h$. In the $15 \mathrm{GHz}$ frequency band, the Tx antenna might be mounted on different heights for various indoor applications. In the typical corridor used in Section 5.2, the Tx height was changed to investigate its influence on channel parameters. To obtain the large-scale fading from the corresponding received power, the effects of small-scale fading are removed by averaging using a 40wavelength sliding window [38]. Based on a log-normal "shadowing" model [35], a path loss model is expressed as follows:

$$
\mathrm{PL}(\mathrm{d})=\operatorname{PL}\left(d_{0}\right)+10 n \log _{10}\left(\frac{d}{d_{0}}\right)+X_{\sigma},
$$

where $\operatorname{PL}\left(d_{0}\right)$ is the intercept value of the path loss model at the reference distance $d_{0}$, which is $1 \mathrm{~m}, n$ is the path loss exponent, and $X_{\sigma}$ is a zero-mean Gaussian distributed random variable with standard deviation of $\sigma_{s}$ describing the random variation about the linear fit. From a large number of simulation results including all the multipath components as in blue curve in Figure 12, it can be seen that the path loss exponent $n$ and the intercept value $d_{0}$ are not influenced by $h$. Based on the Least Squares (LS) fit, the average $n$ and PL $\left(d_{0}\right)$ are 1.93 and $50.4 \mathrm{~dB}$, respectively. The LS fit of using these values of $n$ and $\operatorname{PL}\left(d_{0}\right)$ when $h=1.5 \mathrm{~m}$ is shown in Figure 13 .

An interesting finding is observed here. Although the path loss exponent $n$ and the intercept value $d_{0}$ are not affected by $h$, the standard deviation $\sigma_{s}$ of shadow fading is found to be related to $h$. To state this feature clear, three mathematical formulas are presented in the same way as in [39]:

$$
\begin{aligned}
& \text { Model 1: } \sigma_{s}=a h+b, \\
& \text { Model 2: } \sigma_{s}=\frac{a}{h}+b, \\
& \text { Model 3: } \sigma_{s}=a \log _{10} h+b,
\end{aligned}
$$

TABLE 4: Parameters of three models.

\begin{tabular}{lcccccc}
\hline & \multicolumn{2}{c}{ Model 1 } & \multicolumn{2}{c}{ Model 2 } & \multicolumn{2}{c}{ Model 3 } \\
\hline & $a$ & $b$ & $a$ & $b$ & $a$ & $b$ \\
& -0.975 & 2.699 & 3.553 & -0.741 & -3.957 & 2.266 \\
$R^{2}$ & 0.9594 & \multicolumn{2}{c}{0.9965} & \multicolumn{2}{c}{0.9861} \\
RMSE & 0.087 & \multicolumn{2}{c}{0.025} & \multicolumn{2}{c}{0.051} \\
\hline
\end{tabular}

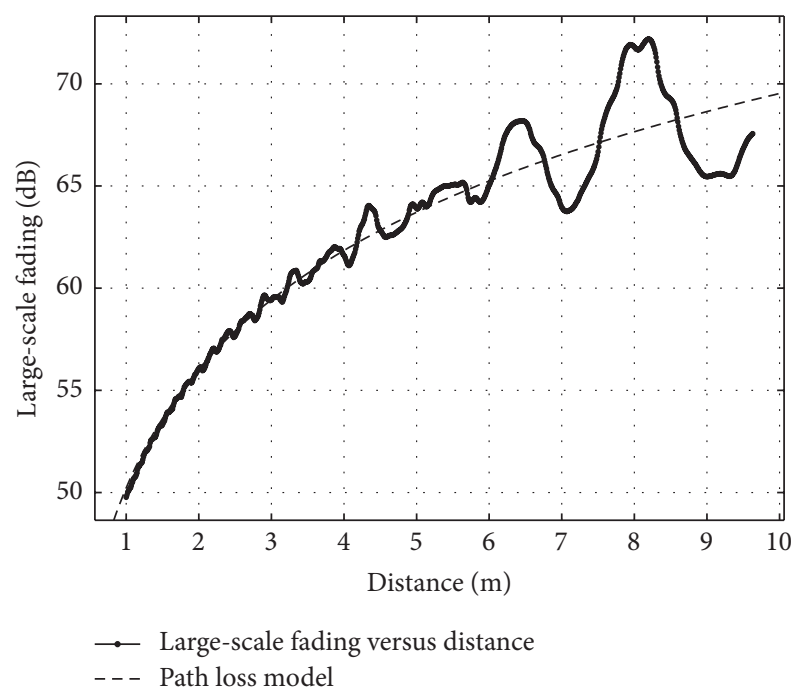

FIGURE 13: LS fit of large-scale fading versus link distance for a typical corridor.

where $h$ is the Tx height and $a$ and $b$ are undetermined coefficients. The fit curves of the three models are shown in Figure 14. According to the fits, the parameters of models are given in Table 4 . The goodness of each fit is evaluated by RMSE and $R^{2}$ :

$$
\begin{aligned}
\text { RMSE } & =\sqrt{\frac{1}{M-1} \sum_{i=1}^{M}\left(y_{i}-\bar{y}\right)^{2}}, \\
R^{2} & =1-\frac{\sum_{i=1}^{M}\left(y_{i}-\widehat{y}_{i}\right)^{2}}{\sum_{i=1}^{M}\left(y_{i}-\bar{y}\right)^{2}},
\end{aligned}
$$

where $y_{i}$ and $\hat{y}_{i}$ are measured value and estimated value, respectively. $\bar{y}$ is mean value of measured data and $M$ is number of samples. RMSE represents the sample standard deviation of the differences between predicted values and observed values, and $R^{2}$ indicates how successful the fit is in describing the variation of the data. A value closer to 0 for RMSE and a value closer to 1 for $R^{2}$ show a better fit of model. With lowest RMSE of 0.025 and highest $R^{2}$ of 0.9965 , Model 2 is found to be the best model to describe the influence of $h$. The standard deviation $\sigma_{s}$ can be written as follows:

$$
\sigma_{s}=\frac{3.553}{h}-0.741,
$$

where units of $h$ are in meters.

According to (11), the Tx located closer to the ceiling of the corridor leads to a smaller fluctuation of path loss. 


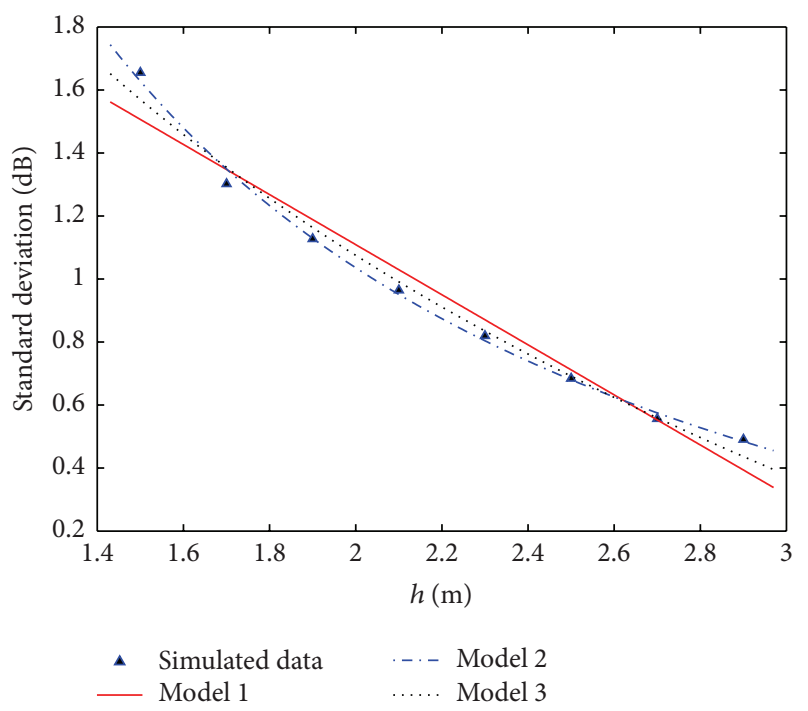

FIGURE 14: The fit curves of three models versus Tx height $h$.

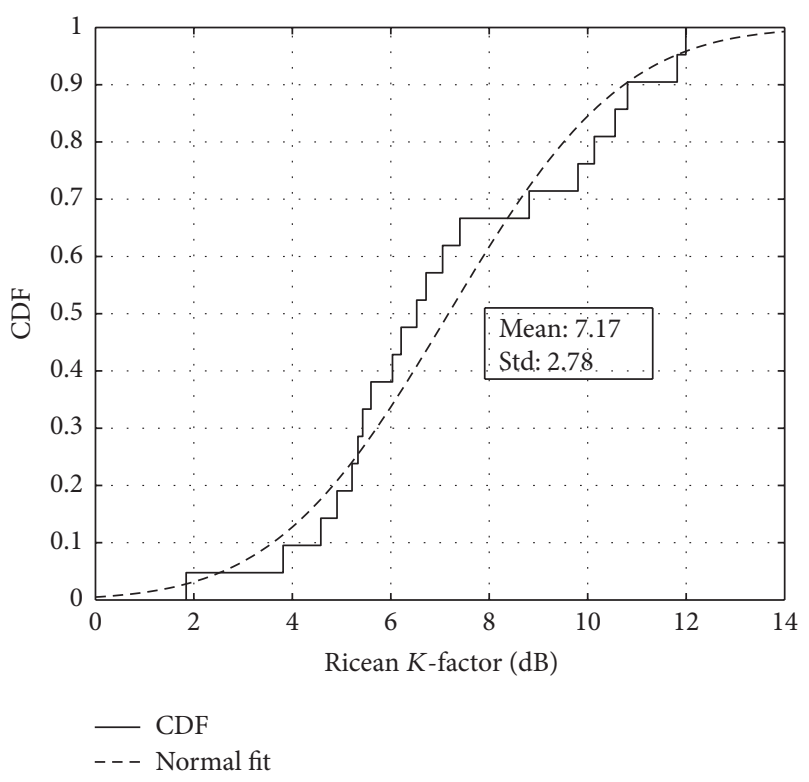

FIgURE 15: CDF of Ricean $K$-factor in the typical corridor.

5.4. Analysis of Small-Scale Fading Properties. In the typical corridor used in Section 5.2, the heights of Tx and $\mathrm{Rx}$ are both $1.5 \mathrm{~m}$. The influences of antennas have been removed. As the large-scale fading data including path loss and shadowing are obtained by using a 40-wavelength sliding window [38], the small-scale fading data can be obtained by removing the large-scale fading from the simulated data. Because of the clear LoS in this case, the amplitudes of small-scale fading are described by a Ricean distribution. The most important parameter of this distribution is Ricean $K$-factor which is vital to the design of wireless communication techniques [40]. In this paper, the $K$-factor is estimated by the momentbased method [41], in the set of 20-wavelength window as a function of distance. In this case, CDF of Ricean $K$-factor and the corresponding fitting with a lognormal distribution are shown in Figure 15. A $K$-factor with a mean value of $7.17 \mathrm{~dB}$ and a standard deviation of $2.78 \mathrm{~dB}$ is observed, which is similar to the results in our previous work [42]. From the simulated results, the fluctuations of $K$-factor always exist at $15 \mathrm{GHz}$ for most of the locations, from almost $5 \mathrm{~dB}$ to $10 \mathrm{~dB}$.

\section{Conclusion}

A measurement campaign for indoor corridor scenarios in the $15 \mathrm{GHz}$ band has been carried out, and a deterministic propagation model with both antenna modeling and propagation modeling was proposed. The model was validated by comparing its values of received power and RMS delay spread to those obtained by measurements. After removing the influence of the directional antennas, the path loss exponent was found to be mainly affected by multiple reflection paths and the scattering paths which also lead to deep fading. Finally, a statistical path loss model incorporating Tx height was proposed based on the generated samples with the proposed deterministic model. It shows that the average path loss exponent is 1.93 and the standard deviation is closely related to Tx height. For different locations, the fluctuations of Ricean $K$-factor always exist, with values of $K$ ranging from almost $5 \mathrm{~dB}$ to $10 \mathrm{~dB}$. Moreover, the limited measurement results were used to validate our ray-optical simulator that is shown to be able to predict the propagation in various indoor corridors in the $15 \mathrm{GHz}$ band.

\section{Competing Interests}

The authors declare that they have no competing interests.

\section{Acknowledgments}

This research is supported by the Fundamental Research Funds for the Central Universities under Grant no. 2015YJS029, the Natural Science Base Research Plan in Shaanxi Province of China under Grant no. 2015JM6320, the NNSF of China under Grant 61501021, and the National Key Technology R\&D Program under Grant 2014BAK02B05.

\section{References}

[1] A. Gohil, H. Modi, and S. K. Patel, "5G technology of mobile communication: a survey," in Proceedings of the International Conference on Intelligent Systems and Signal Processing (ISSP '13), pp. 288-292, Gujarat, India, March 2013.

[2] H. Viswanathan and M. Weldon, "The past, present, and future of mobile communications," Bell Labs Technical Journal, vol. 19, pp. 8-21, 2014.

[3] J. Thompson, X. Ge, H. Wu et al., " $5 \mathrm{G}$ wireless communication systems: prospects and challenges [Guest Editorial]," IEEE Communications Magazine, vol. 52, no. 2, pp. 62-64, 2014.

[4] "5G vision and requirement white papers," 2014, http://www .imt-2020.cn/en.

[5] B. Ai, K. Guan, M. Rupp et al., "Future railway services-oriented mobile communications network," IEEE Communications Magazine, vol. 53, no. 10, pp. 78-85, 2015. 
[6] J. Karjalainen, M. Nekovee, H. Benn, W. Kim, J. Park, and H. Sungsoo, "Challenges and opportunities of mm-wave communication in 5G networks," in Proceedings of the 9th International Conference on Cognitive Radio Oriented Wireless Networks (CROWNCOM '14), pp. 372-376, Oulu, Finland, June 2014.

[7] J. G. Andrews, S. Buzzi, W. Choi et al., "What will 5G be?" IEEE Journal on Selected Areas in Communications, vol. 32, no. 6, pp. 1065-1082, 2014.

[8] J. Medbo, K. Borner, K. Haneda et al., "Channel modelling for the fifth generation mobile communications," in Proceedings of the 8th European Conference on Antennas and Propagation (EuCAP '14), pp. 219-223, IEEE, The Hague, The Netherlands, April 2014.

[9] Y. Wang, J. Li, L. Huang, Y. Jing, A. Georgakopoulos, and P. Demestichas, "5G mobile: spectrum broadening to higherfrequency bands to support high data rates," IEEE Vehicular Technology Magazine, vol. 9, no. 3, pp. 39-46, 2014.

[10] G. Butt, B. G. Evans, and M. Richharia, "Narrowband channel statistics from multiband propagation measurements applicable to high elevation angle land-mobile satellite systems," IEEE Journal on Selected Areas in Communications, vol. 10, no. 8, pp. 1219-1226, 1992.

[11] S. Scalise, H. Ernst, and G. Harles, "Measurement and modeling of the land mobile satellite channel at Ku-band," IEEE Transactions on Vehicular Technology, vol. 57, no. 2, pp. 693-703, 2008.

[12] F. Perez-Fontan, N. Jeannin, L. Castanet et al., "Statistical and physical-statistical modeling of the land mobile satellite, LMS, channel at Ku- and Ka-band," in Proceedings of the 5th European Conference on Antennas and Propagation (EUCAP '11), pp. 32373241, Rome, Italy, April 2011.

[13] F. Teschl, V. Hovinen, F. Pérez-Fontán, M. Schönhuber, and R. Prieto-Cerdeira, "Narrow- and wideband land mobile satellite channel statistics for various environments at Ku-band," in Proceedings of the 6th European Conference on Antennas and Propagation (EuCAP '12), pp. 2464-2468, March 2012.

[14] S. Scalise, M. A. V. Castro, A. Jahn, and H. Ernst, "A comparison of the statistical properties of the land mobile satellite channel at $\mathrm{Ku}, \mathrm{Ka}$ and EHF bands," in Proceedings of the IEEE 61st Vehicular Technology Conference (VTC '05), pp. 2687-2691, June 2005.

[15] D. Donald, S. Kumar, and B. Daku, "Measurement of the indoor RF propagation channel at Ku frequency band," in Proceedings of the IEEE Communications, Computers and Power in the Modern Environment (WESCANEX '93), pp. 66-71, Saskatoon, Canada, May 1993.

[16] B. Ai, X. Cheng, T. Kurner et al., "Challenges toward wireless communications for high-speed railway," IEEE Transactions on Intelligent Transportation Systems, vol. 15, no. 5, pp. 2143-2158, 2014.

[17] Y. Li, B. Ai, X. Cheng, S. Lin, and Z. Zhong, "A TDL based non-WSSUS vehicle-to-vehicle channel model," International Journal of Antennas and Propagation, vol. 2013, Article ID 103461, 8 pages, 2013.

[18] A. Chandra, A. Kumar, and P. Chandra, "Comparative study of path losses from propagation measurements at $450 \mathrm{MHz}$, $900 \mathrm{MHz}, 1.35 \mathrm{GHz}$ and $1.89 \mathrm{GHz}$ in the corridors of a multifloor laboratory-cum-office building," in Proceedings of the IEEE VTS 50th Vehicular Technology Conference (VTC'99), pp. 22722276, Amsterdam, The Netherlands, September 1999.

[19] C. Oestges, D. Vanhoenacker-Janvier, and B. Clerckx, "Channel characterization of indoor wireless personal area networks," IEEE Transactions on Antennas and Propagation, vol. 54, no. 11, pp. 3143-3150, 2006.
[20] C. Oestges, B. Clerckx, L. Bollen, and D. Vanhoenacker-Janvier, "Indoor channel characterization of diversity-based nomadic wireless systems," in IEEE Global Telecommunications Conference (GLOBECOM '05), pp. 3418-3422, St Louis, Mo, USA, December 2005.

[21] C. M. Tan, A. R. Nix, and M. A. Beach, "Dynamic spatialtemporal propagation measurement and super-resolution channel characterisation at $5.2 \mathrm{GHz}$ in a corridor environment," in Proceedings of the 56th Vehicular Technology Conference (VTC '02), pp. 797-801, IEEE, September 2002.

[22] N. Moraitis and P. Constantinou, "Measurements and characterization of wideband indoor radio channel at $60 \mathrm{GHz}$," IEEE Transactions on Wireless Communications, vol. 5, no. 4, pp. 880889, 2006.

[23] C.-F. Yang, B.-C. Wu, and C.-J. Ko, "A ray-tracing method for modeling indoor wave propagation and penetration," IEEE Transactions on Antennas and Propagation, vol. 46, no. 6, pp. 907-919, 1998.

[24] N. Sood and C. Sarris, "Realistic ray-tracing based assessment of MIMO performance in indoor environments," in Proceedings of the IEEE Antennas and Propagation Society International Symposium (APSURSI '12), pp. 1-2, Chicago, Ill, USA, July 2012.

[25] O. Katircioglu, H. Isel, O. Ceylan, F. Taraktas, and H. B. Yagci, "Comparing ray tracing, free space path loss and logarithmic distance path loss models in success of indoor localization with RSSI," in Proceedings of the 19th Telecommunications Forum (TELFOR '11), pp. 313-316, Belgrade, Serbia, November 2011.

[26] T. R. Rao, D. Murugesan, S. Ramesh, and V. A. Labay, "Radio channel characteristics in an indoor corridor environment at $60 \mathrm{GHz}$ for wireless networks," in Proceedings of the 5th IEEE International Conference on Advanced Networks and Telecommunication Systems (ANTS '11), pp. 1-5, Bangalore, India, December 2011.

[27] ITU-R Recommendations, ITU-R P. 1407-3, "Multipath propagation and parameterization of its characteristics", 2007.

[28] ITU-R Recommendations, ITU-R P. 1238-7 "Propagation data and prediction methods for the planning of indoor radiocommunication systems and radio local area networks in the frequency range $900 \mathrm{MHz}$ to $100 \mathrm{GHz} " 2012$.

[29] M. Schack, Integrated simulation of communication applications in vehicular environments [Ph.D. thesis], Technische Universitat Braunschweig, Braunschweig, Germany, 2013.

[30] K. Guan, Z. Zhong, B. Ai, and T. Kürner, "Deterministic propagation modeling for the realistic high-speed railway environment," in Proceedings of the IEEE 77th Vehicular Technology Conference (VTC '13), pp. 1-5, June 2013.

[31] H. Wei, Z. Zhong, K. Guan et al., "Path loss models in viaduct and plain scenarios of the high-speed railway," in Proceedings of the 5th International ICST Conference on Communications and Networking (CHINACOM '10), pp. 1-5, Beijing, China, August 2010.

[32] J. W. McKown and R. L. Hamilton Jr., "Ray tracing as a design tool for radio networks," IEEE Network, vol. 5, no. 6, pp. 27-30, 1991.

[33] R. C. Jones, "A new calculus for the treatment of optical systemsiii the sohncke theory of optical activity," Journal of the Optical Society of America, vol. 31, no. 7, pp. 500-503, 1941.

[34] A. Maltsev, R. Maslennikov, A. Lomayev et al., "Polarization Model for $60 \mathrm{GHz}$, IEEE 802.11-09/0431r0, 2009.

[35] T. Rappaport, Wireless Communications Principles and Practice, Prentice-Hall, Englewood Cliffs, NJ, USA, 2nd edition, 2011. 
[36] J. Jemai, P. Eggers, G. Pedersen et al., "On the applicability of deterministic modeling to indoor UWB channels," in Proceedings of the 3rd Workshop on Positioning, Navigation and Communication (WPNC'06), pp. 139-148, Hanover, Germany, 2006.

[37] A. F. Molisch and M. Steinbauer, "Condensed parameters for characterizing wideband mobile radio channels," International Journal of Wireless Information Networks, vol. 6, no. 3, pp. 133$154,1999$.

[38] W. C. Lee, "Estimate of local average power of a mobile radio signal," IEEE Transactions on Vehicular Technology, vol. 34, no. 1, pp. 22-27, 1985.

[39] R. He, Z. Zhong, B. Ai, and J. Ding, "An empirical path loss model and fading analysis for high-speed railway viaduct scenarios," IEEE Antennas and Wireless Propagation Letters, vol. 10, pp. 808-812, 2011.

[40] L. J. Greenstein, S. S. Ghassemzadeh, V. Erceg, and D. G. Michelson, "Ricean $\mathrm{K}$-factors in narrow-band fixed wireless channels: theory, experiments, and statistical models," IEEE Transactions on Vehicular Technology, vol. 58, no. 8, pp. 40004012, 2009.

[41] A. Abdi, C. Tepedelenlioglu, M. Kaveh, and G. Giannakis, "On the estimation of the $\mathrm{K}$ parameter for the rice fading distribution," IEEE Communications Letters, vol. 5, no. 3, pp. 92-94, 2001.

[42] Q. Wang, B. Ai, K. Guan, Y. Li, and Z. Zhong, "Ray-based analysis of small-scale fading for indoor corridor scenarios at $15 \mathrm{GHz}$," in Proceedings of the Asia-Pacific Symposium on Electromagnetic Compatibility (APEMC '15), pp. 181-184, IEEE, Taipei, Taiwan, May 2015. 


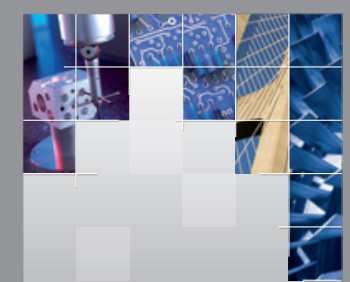

\section{Enfincering}
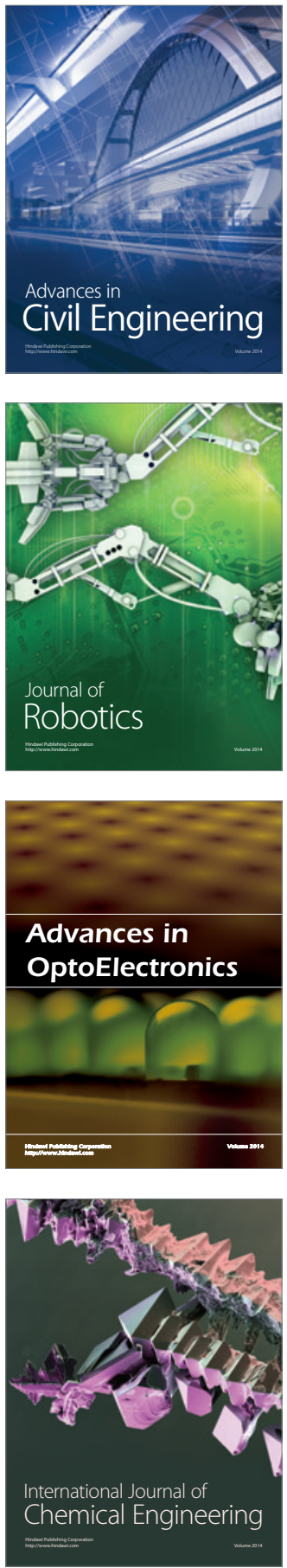

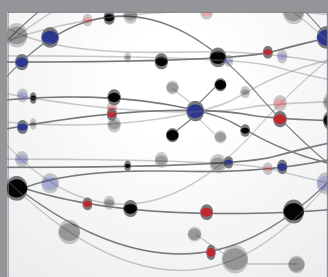

The Scientific World Journal

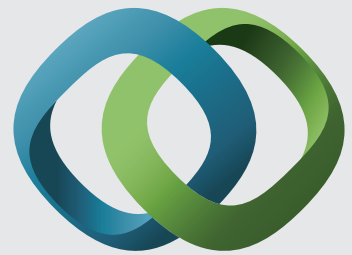

\section{Hindawi}

Submit your manuscripts at

http://www.hindawi.com
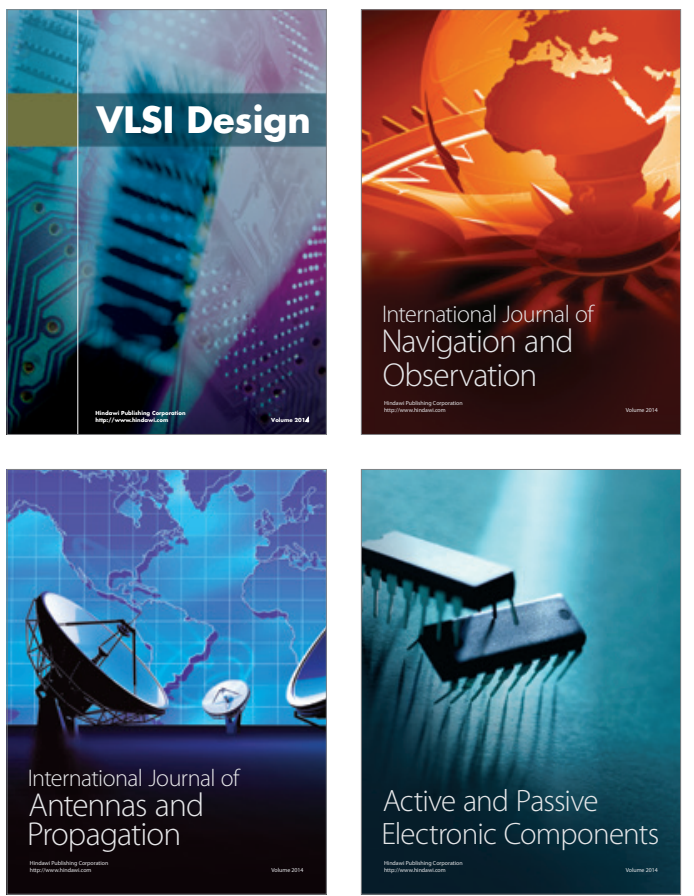
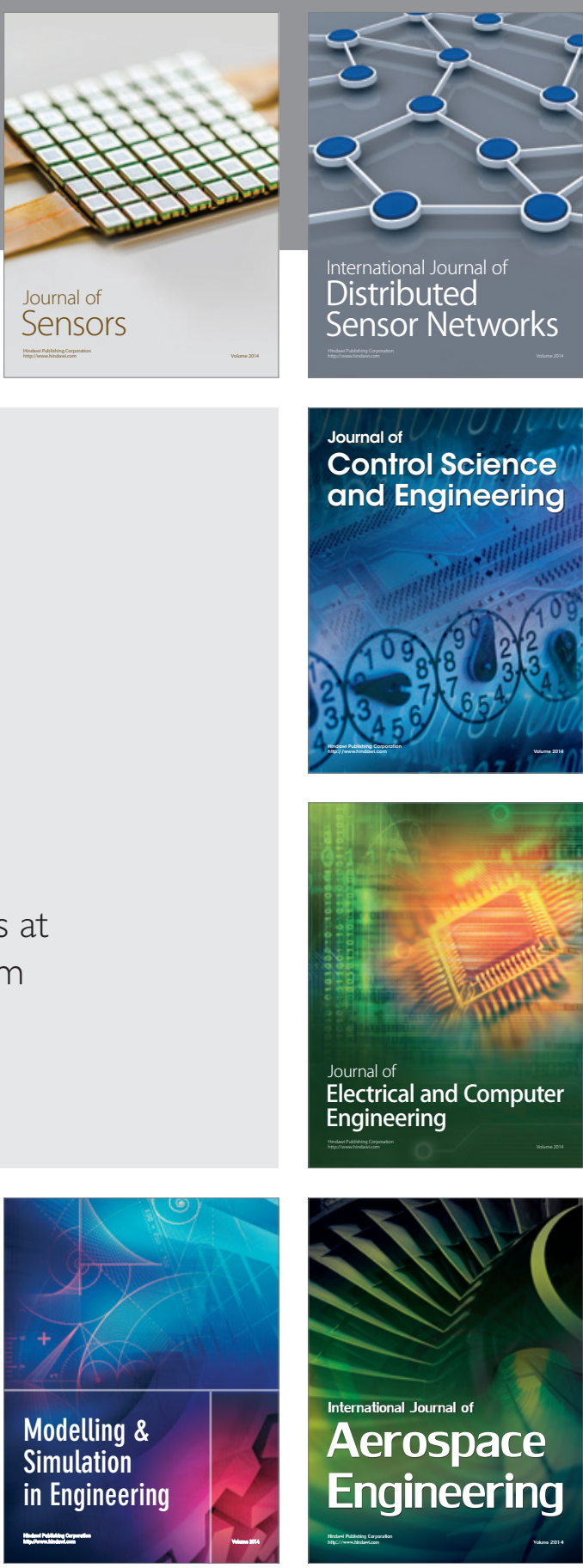

International Journal of

Distributed

Sensor Networks

Journal of

Control Science

and Engineering
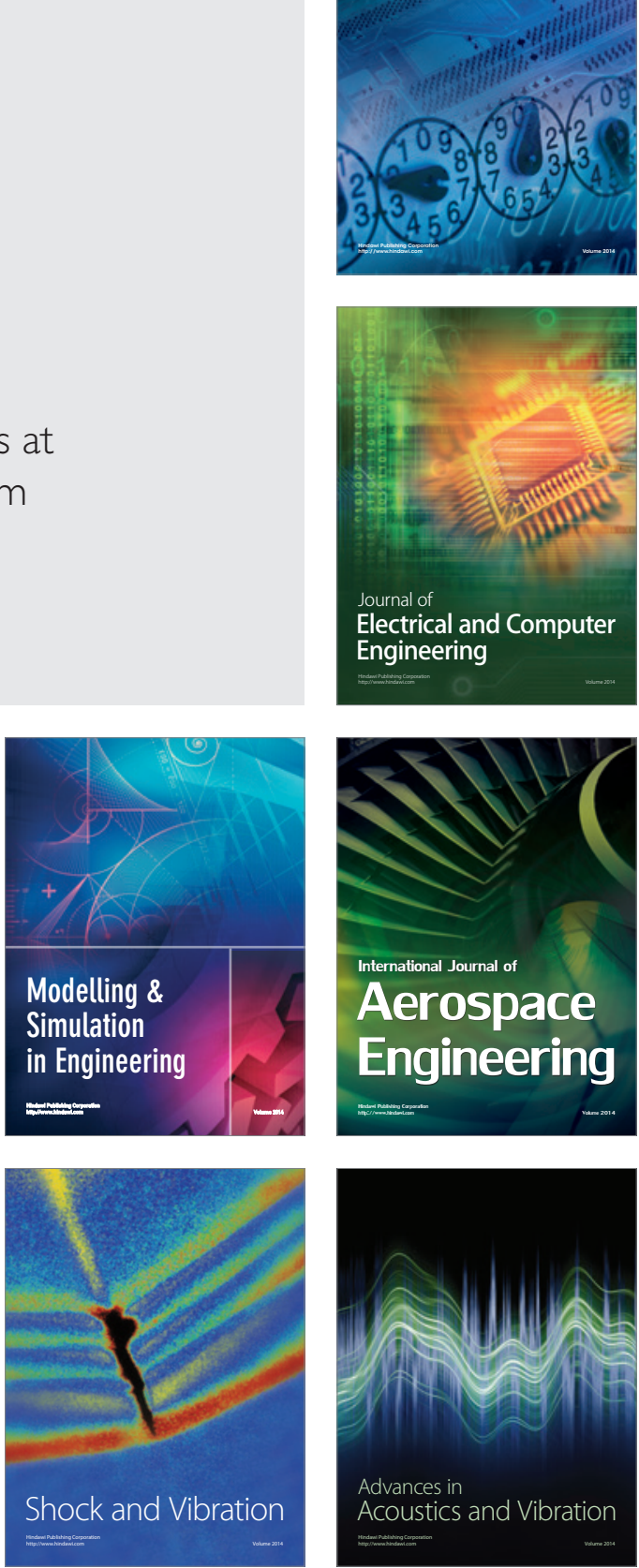